\title{
PLATE BUCKLING INCLUDING EFFECTS OF SHEAR DEFORMATION AND PLATE BENDING CURVATURES USING THE BOUNDARY ELEMENT METHOD
}

\author{
LEANDRO PALERMO JR. ${ }^{1}$, VITOR CAUE GOMES ${ }^{1} \&$ LUIZ CARLOS WROBEL ${ }^{2}$ \\ ${ }^{1}$ School of Civil Engineering, Architecture and Urban Design, State University of Campinas, Brazil \\ ${ }^{2}$ Department of Civil Engineering, Pontifical Catholic University of Rio de Janeiro, Brazil
}

\begin{abstract}
In this paper, the plate bending curvature was included in the geometrical non-linearity (GNL) effect beyond the deflection derivatives to perform plate buckling analyses. The boundary element method (BEM) was adopted and the formulation employed two integrals related to the GNL effect, with one computed on the boundary and the other on the domain. The eigenvalue problem was solved with the inverse iteration method. Results obtained with different boundary conditions were compared to values in the literature.
\end{abstract}

Keywords: plate buckling, Mindlin plate, Reissner plate, bucking parameter, tangential differential operator.

\section{INTRODUCTION}

In-plane forces affect the plate bending behavior when the deflection surface is considered in the plate equilibrium. The problem is geometrically nonlinear in the case of large deflections, when the stretching and the bending of the plate become coupled [1]. Buckling analysis is one of the ways to evaluate the effect of in-plane forces when deflections remain small and the in-plane forces can be related to in-plane tractions. Timoshenko and Woinowsky-Krieger [1] presented the equilibrium equation for the classical bending model with the geometric non-linearity (GNL) effect containing the derivatives of the plate deflection weighted by in-plane forces, which is used in buckling analyses. The inclusion of the effect of the shear deformation in the bending model improves the accuracy of the plate stresses computation [2] or the dynamical behavior of the plate [3]. The buckling load values present significant changes according to plate thickness values beyond the flexural rigidity when the effect of shear deformation is included in the bending model [4], with reference to results obtained with the classical model even considering the same GNL effect presented by Timoshenko in both models. Questions arise on the effect of shear deformation in the GNL when buckling analyses for moderately thick plates are performed. Dawe and Roufaeil [5] discussed the plate buckling analyses considering the effect of shear deformation following the first study presented by Herrmann and Armenakas [6]. The inclusion of the curvatures (the first derivative of rotations) beyond the deflection derivatives in the GNL effect was the main point in the discussion. Sun [7] presented a detailed and comprehensive study using the equations of motion for the Timoshenko beam with the curvature included in the GNL effect beyond the deflection derivative. Sun considered the Trefftz and the Biot theory in the analyses and showed the buckling loads were reduced in the range of intermediate wavelengths, i.e., when the shear deformation is pronounced. Brunelle and Robertson [8] presented two ways to obtain the differential equations for Mindlin plates under a general state of non-uniform initial stress considering the curvatures and the deflection derivatives in the GNL effect. Mizusawa [9] showed the effect of curvatures was greater for certain types of boundary conditions, whereas it was not significant for others when the derivatives of deflection would be enough for the analyses. Smith [10] proposed a finite element 
formulation including both derivatives on displacements (deflection and rotations) in the buckling of thick plates. Doong [11] and Matsunaga [12] studied improvements to the model representing the effect of shear deformation in plate bending, where the buckling analyses considered the derivatives of rotations and deflections combined with a high-order theory representing the effect of shear deformation.

The boundary element formulation for buckling analyses derived in this study employs two integrals containing the GNL effect, with one computed on the domain and the other computed on the boundary. The first derivatives of the deflection and rotations were used in the kernels of integrals related to the GNL effect, and no relation was required for the derivatives of in-plane forces. The natural conditions of the buckling problem were considered and related to boundary integrals containing the GNL effect in case of free edges or when the deflection and/or rotations were released for certain types of boundary conditions, i.e., the hard or the soft condition. The present formulation contains the general GNL effect with reference to that presented in [4], where only the first derivatives of the deflection were considered. The numerical implementation employed quadratic shape functions to approximate displacements (deflections and rotations), distributed shears, and moments in the boundary elements whereas constant elements were used to discretize both integrals related to the GNL effect. Constant elements were the lower type of element to evaluate the behavior of the formulation containing the GNL effect. An algebraic manipulation using both integrals with the GNL effect was carried out to perform integrations only on the sides of cells inside the domain in problems with known displacements (deflection and rotations) on the plate boundary. The inverse iteration and Rayleigh quotient were used to compute the lowest eigenvalues with the corresponding eigenvectors. The changes in the value of the buckling parameter according to the plate thickness were compared to values available in the literature.

\section{BOUNDARY INTEGRAL EQUATIONS}

The constitutive equations are written next with a unified notation for the Reissner and Mindlin bending models. The Latin indices take on values $\{1,2$ and 3$\}$ and Greek indices take on values $\{1,2\}$.

$$
\begin{gathered}
M_{\alpha \beta}=D \frac{(1-v)}{2}\left(u_{\alpha, \beta}+u_{\beta, \alpha}+\frac{2 v}{1-v} u_{\gamma, \gamma} \delta_{\alpha \beta}\right)+\delta_{\alpha \beta} q R E, \\
Q_{\alpha}=D \frac{(1-v)}{2} \lambda^{2}\left(u_{\alpha}+u_{3, \alpha}\right),
\end{gathered}
$$

with

$$
D=\frac{E h^{3}}{12\left(1-v^{2}\right)} \quad \lambda^{2}=12 \frac{\kappa^{2}}{h^{2}} ; \quad R E=\frac{v}{\lambda^{2}(1-v)},
$$

where $\mathrm{u}_{\alpha}$ is the plate rotation in direction $\alpha$, and $\mathrm{u}_{3}$ is the plate deflection, $\mathrm{D}$ is the flexural rigidity, $\mathrm{h}$ is the plate thickness, $v$ is Poisson's ratio, $\mathrm{q}$ is the distributed load on the plate domain and $\delta_{\alpha \beta}$ is the Kronecker delta. The product qRE in eqn (1) corresponds to the linearly weighted average effect of the normal stress component in the thickness direction, which should be considered in the Reissner model [2] but not in the Mindlin model [3] $(\mathrm{RE}=0)$. This term is null in buckling problems because the distributed load $\mathrm{q}$ is equal to zero. The shear parameter is equal to $5 / 6$ and $\pi^{2} / 12$ for the Reissner and the Mindlin model, 
respectively, and it is the only difference introduced according to the model employed in the analysis.

The natural conditions and the equilibrium equations for the problem can be obtained with the calculus of variations [13], [14]. The energy functional of the plate is given by:

$$
\begin{gathered}
\Pi=\int_{\Omega}\left\{\frac{D(1-v)}{4}\left[u_{\alpha, \beta}^{2}+u_{\alpha, \beta} u_{\beta, \alpha}+\frac{2 v}{(1-v)} u_{\gamma, \gamma}^{2}+\lambda^{2}\left(u_{\alpha}+u_{3, \alpha}\right)^{2}\right]\right\} d \Omega+\cdots \\
+\int_{\Omega} \frac{h^{2}}{24}\left(N_{\alpha \beta} u_{\gamma, \alpha} u_{\gamma, \beta}\right) d \Omega+\int_{\Omega} \frac{1}{2}\left(N_{\alpha \beta} u_{3, \alpha} u_{3, \beta}\right) d \Omega-\int_{\Gamma_{f}}\left(P w+E M_{\alpha} u_{\alpha}\right) d \Gamma .
\end{gathered}
$$

The energy functional of the plate was written in the complete form without the distributed load in eqn (3). The first integral (domain integral) is the strain energy whereas the GNL effect appeared in subsequent integrals containing the in-plane forces $\left(\mathrm{N}_{\alpha \beta}\right)$. The last integral is the potential energy of the external loads. $\mathrm{EM}_{1}$ and $\mathrm{EM}_{2}$ are couples in directions 1,2 and $\mathrm{P}$ is the out-of-plane load distributed on a portion of the boundary $\left(\Gamma_{\mathrm{f}}\right)$. The displacements $\left(\mathrm{u}_{1}, \mathrm{u}_{2}\right.$ and $\left.\mathrm{u}_{3}\right)$ are not prescribed on the portion of the boundary line $\Gamma_{\mathrm{f}}$. The energy functional of the plate can be written as a general function to be minimized with the calculus of variations:

$$
\Pi=\int_{\Omega} F\left(u_{1}, u_{2}, u_{3}, u_{1,1}, u_{2,1}, u_{3,1}, u_{1,2}, u_{2,2}, u_{3,2}\right) d \Omega
$$

The Euler equations obtained from the minimization of eqn (4) are given by:

$$
\frac{\partial F}{\partial u_{i}}-\frac{\partial}{\partial x_{\alpha}}\left(\frac{\partial F}{\partial u_{i, \alpha}}\right)=0_{(i=1,2,3)} .
$$

The equilibrium equations are obtained when the constitutive equations are introduced in the resultant expressions from the application of Euler equations:

$$
\begin{gathered}
M_{\gamma \beta, \beta}-Q_{\gamma}+\frac{h^{2}}{12} \frac{\partial}{\partial x_{\alpha}}\left(N_{\alpha \beta} \frac{\partial u_{\gamma}}{\partial x_{\beta}}\right)=0, \\
Q_{\alpha, \alpha}+\frac{\partial}{\partial x_{\alpha}}\left(N_{\alpha \beta} \frac{\partial u_{3}}{\partial x_{\beta}}\right)=0
\end{gathered}
$$

The natural conditions introduce the requirements on the boundary portion $\left(\Gamma_{\mathrm{f}}\right)$ with not prescribed displacements where the variations on displacements are not null $\left(\delta u_{i} \neq 0\right)$ :

$$
\begin{gathered}
\left(\frac{\partial F}{\partial u_{i, \alpha}} n_{\alpha}\right) \delta u_{i}=0 \stackrel{\text { yields }}{\longrightarrow} \frac{\partial F}{\partial u_{i, \alpha}} n_{\alpha}=0, \\
t_{\gamma}=E M_{\gamma}-\frac{h^{2}}{12}\left(n_{\alpha} N_{\alpha \beta} u_{\gamma, \beta}\right), \\
t_{3}=P-n_{\alpha} N_{\alpha \beta} u_{3, \beta} .
\end{gathered}
$$


The constitutive equations were used to obtain $\mathrm{t}_{\alpha}\left(\mathrm{t}_{\alpha}=\mathrm{M}_{\alpha \beta} \cdot \mathrm{n}_{\beta}\right)$ and $\mathrm{t}_{3}\left(\mathrm{t}_{3}=\mathrm{Q}_{\alpha} \cdot \mathrm{n}_{\alpha}\right)$, respectively, in the natural conditions.

The general form of the displacement boundary integral equations (DBIEs) with an additional domain integral containing the GNL effect is written next with the notation proposed by Weeën:

$$
\begin{gathered}
\frac{1}{2} C_{i j}\left(x^{\prime}\right) u_{j}\left(x^{\prime}\right)+\int_{\Gamma}\left[T_{i j}\left(x^{\prime}, x\right) u_{j}(x)-U_{i j}\left(x^{\prime}, x\right) t_{j}(x)\right] d \Gamma(x)=\cdots \\
=\iint_{\Omega}\left\{U_{i 3}\left(x^{\prime}, X\right)\left[\frac{\partial}{\partial X_{\alpha}}\left(N_{\alpha \beta} \frac{\partial u_{3}}{\partial X_{\beta}}\right)\right]+U_{i \gamma}\left(x^{\prime}, X\right) \frac{h^{2}}{12}\left[\frac{\partial}{\partial X_{\alpha}}\left(N_{\alpha \beta} \frac{\partial u_{\gamma}}{\partial X_{\beta}}\right)\right]\right\} d \Omega(X) .
\end{gathered}
$$

in which $\mathrm{C}_{\mathrm{ij}}$ is an element of the matrix $\mathrm{C}$ related to the boundary at the source point, which becomes the identity matrix when a smooth boundary is considered, $U_{i j}$ represents the rotation $(j=1,2)$ or the deflection $(j=3)$ due to a unit couple $(i=1,2)$ or a unit point force $(i=3)$, respectively, $T_{i j}$ represents the moment $(j=1,2)$ or the shear $(j=3)$ due to a unit couple $(i=1,2)$ or a unit point force $(i=3)$, respectively.

It is well known in nonlinear analyses of beams or plates [1], [15] that the natural condition is introduced for each generalized force $t_{i}$ corresponding to the displacement not prescribed. According to eqns (7) and (8), the GNL effect should be introduced when the deflection and/or rotations is/are not prescribed on the boundary portion.

The term related to the GNL effect in eqns (5), (6) or (9) can be simplified with the equilibrium equations for in-plane forces $\left(\mathrm{N}_{\alpha \beta, \alpha}=0\right)$. The second derivatives of the displacements (rotations or deflections) result from the simplification, as shown in several studies in the literature. The equilibrium equations for in-plane forces were not used here as done in [4] but an algebraic manipulation with the divergence theorem was done in the domain integral related to GNL effect in eqn (9), i.e.:

$$
\begin{gathered}
\iint_{\Omega}\left\{U_{i 3}\left(x^{\prime}, X\right)\left[\frac{\partial}{\partial X_{\alpha}}\left(N_{\alpha \beta} \frac{\partial u_{3}}{\partial X_{\beta}}\right)\right]+U_{i \gamma}\left(x^{\prime}, X\right) \frac{h^{2}}{12}\left[\frac{\partial}{\partial X_{\alpha}}\left(N_{\alpha \beta} \frac{\partial u_{\gamma}}{\partial X_{\beta}}\right)\right]\right\} d \Omega(X)=\cdots \\
\int_{\Gamma}\left[U_{i 3}\left(x^{\prime}, x\right) n_{\alpha}(x) N_{\alpha \beta}(x) u_{3, \beta}(x)+\frac{h^{2}}{12} U_{i \gamma}\left(x^{\prime}, x\right) n_{\alpha}(x) N_{\alpha \beta}(x) u_{\gamma, \beta}(x)\right] d \Gamma(x)+\cdots \\
-\iint_{\Omega}\left[U_{i 3, \alpha}\left(x^{\prime}, X\right) N_{\alpha \beta}(X) u_{3, \beta}(X)+\frac{h^{2}}{12} U_{i \gamma, \alpha}\left(x^{\prime}, X\right) N_{\alpha \beta}(X) u_{\gamma, \beta}(X)\right] d \Omega(X) .
\end{gathered}
$$

Two integrals containing the GNL effect result from the algebraic manipulation with the divergence theorem, where one is computed on the boundary and the other on the domain. Despite the increase in the number of integrals with the GNL effect, the first derivatives of the deflection and rotations were only necessary in two integrals and the equilibrium equations for in-plane forces were not required. The final DBIE is given by: 


$$
\begin{gathered}
\frac{1}{2} C_{i j}\left(x^{\prime}\right) u_{j}\left(x^{\prime}\right)+\int_{\Gamma}\left[T_{i j}\left(x^{\prime}, x\right) u_{j}(x)-U_{i j}\left(x^{\prime}, x\right) t_{j}(x)\right] d \Gamma(x)=\cdots \\
=\int_{\Gamma}\left[U_{i 3}\left(x^{\prime}, x\right) n_{\alpha}(x) N_{\alpha \beta}(x) u_{3, \beta}(x)+\frac{h^{2}}{12} U_{i \gamma}\left(x^{\prime}, x\right) n_{\alpha}(x) N_{\alpha \beta}(x) u_{\gamma, \beta}(x)\right] d \Gamma(x)+\cdots \\
-\iint_{\Omega}\left[U_{i 3, \alpha}\left(x^{\prime}, X\right) N_{\alpha \beta}(X) u_{3, \beta}(X)+\frac{h^{2}}{12} U_{i \gamma, \alpha}\left(x^{\prime}, X\right) N_{\alpha \beta}(X) u_{\gamma, \beta}(X)\right] d \Omega(X)
\end{gathered}
$$

The boundary integral containing the GNL effect can be related to natural conditions given by eqns (7) and (8) when the boundary portion has the deflection and rotations not prescribed. This can be shown by assuming the boundary $\Gamma$ split into two portions: $\Gamma_{\mathrm{p}}$ and $\Gamma_{\mathrm{f}}$ where displacements are known (prescribed) and unknown (not prescribed or free), respectively.

$$
\begin{gathered}
\frac{1}{2} C_{i j} u_{j}+\int_{\Gamma_{f}} T_{i j} u_{j} d \Gamma-\int_{\Gamma_{p}} U_{i j} t_{j} d \Gamma=\int_{\Gamma_{f}} U_{i \alpha} E M_{\alpha} d \Gamma+\int_{\Gamma_{f}} U_{i 3} P d \Gamma+\cdots \\
-\int_{\Gamma_{p}} T_{i j} u_{j} d \Gamma-\iint_{\Omega}\left[U_{i 3, \alpha} N_{\alpha \beta} u_{3, \beta}+\frac{h^{2}}{12} U_{i \gamma, \alpha} N_{\alpha \beta} u_{\gamma, \beta}\right] d \Omega+\cdots \\
\quad+\int_{\Gamma_{p}}\left[U_{i 3} n_{\alpha} N_{\alpha \beta} u_{3, \beta}+\frac{h^{2}}{12} U_{i \gamma} n_{\alpha} N_{\alpha \beta} u_{\gamma, \beta}\right] d \Gamma .
\end{gathered}
$$

The left-hand side of eqn (11) contains the unknowns, i.e., displacements on $\Gamma_{\mathrm{f}}$ and forces on $\Gamma_{\mathrm{p}}$. The loads on the boundary portion $\Gamma_{\mathrm{f}}$ were introduced in the right hand side according to natural conditions shown in eqns (7) and (8). A simplification can be done on the boundary portion $\Gamma_{\mathrm{f}}$ due to opposite signals of the natural condition and the boundary integral with the GNL effect, which only needs to be computed on the boundary portion with prescribed displacements $\left(\Gamma_{\mathrm{p}}\right)$ as the result.

The gradient of displacements is required in the DBIE for the buckling problem (eqn 10) to introduce the GNL effect. The BIE for the gradient of displacements at an internal point is obtained by differentiating the eqn (10) with respect to the coordinates of the source point $\left(\mathrm{X}^{\prime}\right)$. The result is next written in terms of differentiation of the field point coordinates and using the tangential differential operator [16].

$$
\begin{aligned}
u_{i, \gamma}\left(X^{\prime}\right) & =\int_{\Gamma}\left\{M_{i \alpha \beta}\left(X^{\prime}, x\right) D_{\gamma \alpha}\left[u_{\beta}(x)\right]+n_{\gamma}(x) Q_{i \beta}\left(X^{\prime}, x\right) u_{\beta}(x)\right\} d \Gamma(x)+\cdots \\
& +\int_{\Gamma}\left\{Q_{i \beta}\left(X^{\prime}, x\right) D_{\gamma \beta}\left[u_{3}(x)\right]-U_{i j, \gamma}\left(X^{\prime}, x\right) t_{j}(x)\right\} d \Gamma(x)+\cdots
\end{aligned}
$$




$$
\begin{aligned}
& +\int_{\Gamma}\left[U_{i 3, \gamma}\left(x^{\prime}, x\right) n_{\alpha}(x) N_{\alpha \beta}(x) u_{3, \beta}(x)+\frac{h^{2}}{12} U_{i \rho, \gamma}\left(x^{\prime}, x\right) n_{\alpha}(x) N_{\alpha \beta}(x) u_{\rho, \beta}(x)\right] d \Gamma(x)+\cdots \\
& -\iint_{\Omega}\left[U_{i 3, \alpha \gamma}\left(x^{\prime}, X\right) N_{\alpha \beta}(X) u_{3, \beta}(X)+\frac{h^{2}}{12} U_{i \rho, \alpha \gamma}\left(x^{\prime}, X\right) N_{\alpha \beta}(X) u_{\rho, \beta}(X)\right] d \Omega(X),
\end{aligned}
$$

with

$$
D_{\alpha \beta}[f(x)]=n_{\alpha}(x) f_{, \beta}(x)-n_{\beta}(x) f_{, \alpha}(x)
$$

\section{THE NUMERICAL IMPLEMENTATION}

In the formulation described in this paper, quadratic shape functions for isoparametric boundary elements were employed with collocation points always placed on the boundary. The same mapping function was used for conformal and non-conformal interpolations, i.e., nodes at ends of quadratic elements remain at ends when discontinuous elements were employed. The collocation points were placed at nodes in case of continuous elements and at positions $(-0.67,0.0,+0.67)$, in the range $(-1,1)$, in case of discontinuous elements, i.e., the collocation points were shifted inside the element at the corresponding end where the discontinuity exists. Singularity subtraction [17] and the transformation of variable technique [18] were employed for the Cauchy and the weak type of singularity, respectively, when integrations were performed on elements containing the collocation points. The standard Gauss-Legendre scheme was employed for integrations on elements (or side of the cell) not containing the collocation points. Rectangular cells were used to discretize the domain integral related to the GNL effect. The derivatives of the displacements (deflection and rotation) at the center of the cell were assumed constant on the cell. This assumption allowed the use of the divergence theorem to convert the domain integral into equivalent boundary integrals performed on sides of the cell. This strategy led to a simplification on the use of integrals containing the GNL effect because they have opposite signals as done in [4]. The GNL effect was computed from integrations performed on sides of cells inside the domain in all problems. The boundary condition is required to include the integrals with the GNL effect computed on sides of the cell on the plate boundary:

1. When the displacements are prescribed on the whole boundary (like a clamped plate on all sides), the GNL effect was not computed from integrations performed on sides of cells on the plate boundary.

2. When the displacements are not prescribed on the boundary portion of the plate $\left(\Gamma_{\mathrm{f}}\right)$, the GNL effect was computed from integrations performed on sides of cells on the boundary portion $\Gamma_{\mathrm{f}}$.

3. In the case of a hard or soft boundary condition, when the deflection is prescribed and the tangential rotation or both rotations is/are not prescribed, the corresponding GNL effect related to curvatures is/are computed on the boundary portion related to the hard or soft condition.

The basic inverse iteration and the Rayleigh quotient were used to perform the eigenvalue analysis [19], i.e.: 


$$
\begin{gathered}
A x^{(k+1)}=B x^{k}, \\
\lambda_{k}=\frac{\left(x^{(k+1)}, x^{k}\right)}{\left(x^{(k+1)}, x^{(k+1)}\right)} .
\end{gathered}
$$

The basic inverse iteration procedure is very efficient to compute the lower eigenvalues with corresponding eigenvectors [19]. The discretized forms of eqns (10) and (12) were used instead of eqn (13) as done in [4]. Starting with an eigenvector $\mathrm{x}^{1}$ with elements equal to 1.0 , the values of the displacements and tractions at the boundary nodes are found with eqn (10); these values are introduced in eqn (12) to obtain the gradient of the displacements (elements of the eigenvector $\mathrm{x}^{2}$ ), and the lowest eigenvalue at the first iteration step was obtained by using eqn (14). The iteration procedure continued until the absolute difference between values of successive eigenvalues was less than $10^{-8}$. The proof of convergence for the lower eigenvalues can be found in [19].

\section{NUMERICAL EXAMPLES}

The Young modulus (E) was $206.9 \mathrm{Gpa}$, the Poisson ratio (v) was 0.3. The buckling parameter $\mathrm{k}$ is a non-dimensional value related to the critical load of the plate $\left(\mathrm{N}_{\mathrm{cr}}\right)$, the length of the plate side (a) and the flexural rigidity (D), which is obtained according to following expression:

$$
k=\frac{a^{2} N_{c r}}{\pi^{2} D}
$$

The buckling parameter $\mathrm{k}$ was obtained according to the following boundary conditions: $\mathrm{S}=$ simply supported edge, $\mathrm{C}=$ clamped edge and $\mathrm{F}=$ free edge.

The results obtained were compared to those presented by Mizusawa [9], which were not different to those presented by Dawe and Roufaeil in [5], but more types of boundary conditions were studied in [9]. The Spline Strip Method was used in [9] and the simply supported boundary condition employed the hard restraint condition (tangential rotation is restrained). Results presented in Tables 1 and 2 used 128 quadratic boundary elements (260 nodes) and 256 constant cells as done in [4].

The differences in the buckling parameter obtained with the GNL effect using only deflection derivatives to those obtained with the present formulation were included in the last row of Tables 1 and 2. The results agreed with the comment on the effect of boundary condition done by Mizusawa in [9]. Results in Table 2 were not significantly changed when the curvatures were included in the GNL effect even for the highest thickness when the maximum difference to [4] was $5.02 \%$. On the other hand, results with curvatures included in Table 1 were significant for higher thicknesses, as shown by Sun on buckling analyses for Timoshenko's beam in [7].

\section{CONCLUSIONS}

Results obtained for buckling analyses with BEM and including curvatures in the GNL effect agreed to those in the literature. The effect of curvatures was greater for the highest thickness in the BEM formulation with reference to those in [9]. The first derivatives of displacements (deflection and rotations) were used in the present formulation with the same algebraic manipulation done in [4] where only deflection derivatives were employed. Furthermore, the $\mathrm{BIE}$ for the gradient of displacements employed the tangential differential operator to reduce 
Table 1: Buckling parameter $(\mathrm{k})$ of the first critical load of square plates under uniaxial inplane loading. Effect of curvatures relevant.

\begin{tabular}{|c|c|c|c|c|c|c|}
\hline Type & $\mathrm{h} / \mathrm{a}$ & [4] & [9] & $\begin{array}{l}\text { This } \\
\text { work }\end{array}$ & $\begin{array}{l}\text { Diff. to } \\
\text { [9] } \\
(\%)\end{array}$ & $\begin{array}{l}\text { Diff. to } \\
{[4]} \\
(\%)\end{array}$ \\
\hline \multirow[t]{2}{*}{ 1) $\mathrm{SSSS}$} & 0.001 & 4.0128 & 4.0 & 4.0127 & 0.32 & 0.00 \\
\hline & 0.010 & 4.0105 & 3.997 & 4.0088 & 0.29 & -0.04 \\
\hline \multirow{3}{*}{ 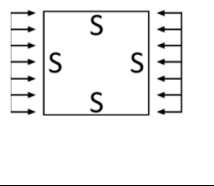 } & 0.050 & 3.9561 & 3.928 & 3.9174 & -0.27 & -0.99 \\
\hline & 0.100 & 3.7952 & 3.729 & 3.6638 & -1.78 & -3.59 \\
\hline & 0.200 & 3.2643 & 3.119 & 2.9587 & -5.42 & -10.33 \\
\hline \multirow[t]{2}{*}{ 2) $\mathrm{SSSC}$} & 0.001 & 4.8707 & 4.8470 & 4.8707 & 0.49 & 0.00 \\
\hline & 0.010 & 4.8666 & 4.8420 & 4.8633 & 0.44 & -0.07 \\
\hline \multirow{3}{*}{ 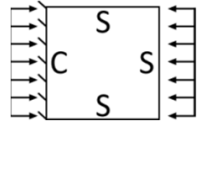 } & 0.050 & 4.7681 & 4.7170 & 4.6940 & -0.49 & -1.58 \\
\hline & 0.100 & 4.4857 & 4.3720 & 4.2541 & -2.77 & -5.44 \\
\hline & 0.200 & 3.6251 & 3.4180 & 3.1927 & -7.06 & -13.54 \\
\hline \multirow[t]{2}{*}{ 3) $\mathrm{CSSS}$} & 0.001 & 5.7597 & 5.7400 & 5.7597 & 0.34 & 0.00 \\
\hline & 0.010 & 5.7538 & 5.7330 & 5.7509 & 0.31 & -0.05 \\
\hline \multirow{3}{*}{ 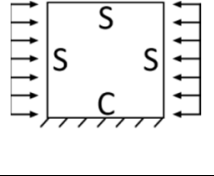 } & 0.050 & 5.6164 & 5.5740 & 5.5488 & -0.45 & -1.22 \\
\hline & 0.100 & 5.2334 & 5.14 & 5.0205 & -2.38 & -4.24 \\
\hline & 0.200 & 4.1468 & 3.8760 & 3.6066 & -7.47 & -14.98 \\
\hline \multirow[t]{2}{*}{ 4) $\mathrm{SCSC}$} & 0.001 & 6.7967 & 6.7430 & 6.7966 & 0.79 & 0.00 \\
\hline & 0.010 & 6.7875 & 6.7310 & 6.7787 & 0.70 & -0.13 \\
\hline \multirow{3}{*}{ 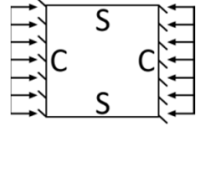 } & 0.050 & 6.5742 & 6.4620 & 6.3827 & -1.24 & -3.00 \\
\hline & 0.100 & 5.9914 & 5.7650 & 5.4702 & -5.39 & -9.53 \\
\hline & 0.200 & 4.4260 & 4.1090 & 3.7064 & -10.86 & -19.42 \\
\hline \multirow[t]{2}{*}{ 5) $\mathrm{CSCS}$} & 0.001 & 7.7540 & 7.692 & 7.7539 & 0.80 & 0.00 \\
\hline & 0.010 & 7.7370 & 7.671 & 7.7284 & 0.74 & -0.11 \\
\hline \multirow{3}{*}{ 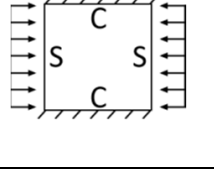 } & 0.050 & 7.3559 & 7.228 & 7.1742 & -0.75 & -2.53 \\
\hline & 0.100 & 6.4138 & 6.178 & 5.9525 & -3.79 & -7.75 \\
\hline & 0.200 & 4.3413 & 4.056 & 3.7733 & -7.49 & -15.05 \\
\hline \multirow{5}{*}{ 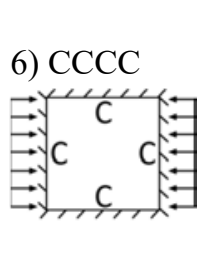 } & 0.001 & 10.1605 & & 10.1603 & & 0.00 \\
\hline & 0.010 & 10.1382 & 10.055 & 10.1225 & 0.67 & -0.16 \\
\hline & 0.050 & 9.6326 & & 9.3115 & & -3.45 \\
\hline & 0.100 & 8.3374 & 8.047 & 7.5670 & -6.34 & -10.18 \\
\hline & 0.200 & 5.3121 & & 4.5041 & & -17.94 \\
\hline
\end{tabular}


Table 2: Buckling parameter $(\mathrm{k})$ of the first critical load of square plates under uniaxial inplane loading. Effect of curvatures not relevant.

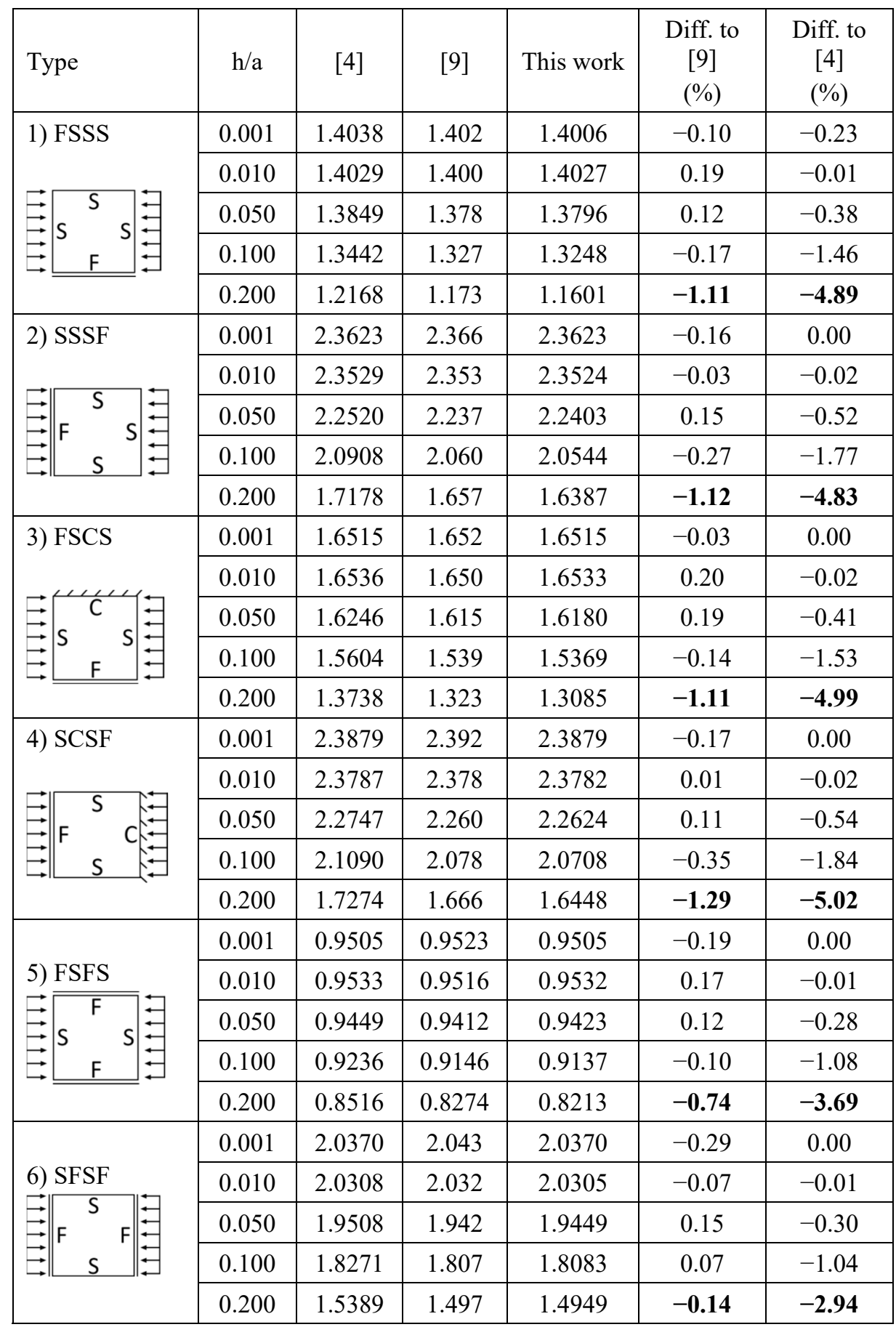


the order of singularity in the kernels of integrals. A BEM formulation for buckling analyses employing only the first derivatives of displacements and including a BIE for the gradient of displacement with singularities reduced were the main numerical features of the present formulation, which present results closer to the literature with a low number of degrees of freedom related to the boundary element analysis.

\section{ACKNOWLEDGEMENT}

The authors are grateful to CAPES (88882.435160/2019-01) for the support in the development of this research.

\section{REFERENCES}

[1] Timoshenko, S.P. \& Woinowsky-Krieger, S., Theory of Plates and Shells, 2nd Ed., McGraw-Hill Book Company: New York, 1959.

[2] Reissner, E., The effect of transverse shear deformation on the bending of elastic plates. Journal of Applied Mechanics, 12(2), pp. A66-A77, 1945.

[3] Mindlin, R.D., Influence of rotatory inertia and shear on flexural motions of isotropic elastic plates. Journal of Applied Mechanics, 18, pp. 18-31, 1951.

[4] Soares, R.A., Jr. \& Palermo, L., Jr., Effect of shear deformation on the buckling parameter of perforated and non-perforated plates studied using the boundary element method. Engineering Analysis with Boundary Elements, 85, pp. 57-69, 2017.

[5] Dawe, D.J. \& Roufaeil, O.L., Buckling of rectangular Mindlin plates. Composite Structures, 15(4), pp. 461-471, 1982.

[6] Herrmann, G. \& Armenakas, A.E., Vibration and stability of plates under initial stress. Transactions of the American Society of Civil Engineers, 127, pp. 458-487, 1962.

[7] Sun, C.T., On the equations for a Timoshenko beam under initial stress. Journal of Applied Mechanics, 39, pp. 282-285, 1972.

[8] Brunelle, E.J. \& Robertson, S.R., Initially stressed Mindlin plates. AIAA Journal, 12, pp. 1036-1045, 1974.

[9] Mizusawa, T., Buckling of rectangular Mindlin plates with tapered thickness by the spline strip method. International Journal of Solids \& Structures, 30(12), pp. 16631677, 1993.

[10] Smith, J.P., Buckling of shear deformable plates using the p-version of the finite element method. Composite Structures, 57(3), pp. 527-532, 1995.

[11] Doong, J.L., Vibration and stability of an initially stressed thick plate according to a high-order deformation theory. Journal of Sound and Vibration, 113, pp. 425-440.

[12] Matsunaga, H., Free vibration and stability of thick elastic plates subjected to in-plane forces. International Journal of Solids and Structures, 31(22), pp. 3113-3124, 1994.

[13] Elsgoltz, L., Ecuaciones Diferenciales y Cálculo Variacional, Editorial Mir: Moscou, 1977.

[14] Katsikadelis, J.T., The Boundary Element Method for Plate Analysis, Elsevier Academic Press, 2014.

[15] Timoshenko, S.P. \& Gere, J.M., Theory of Elastic Stability, 2nd Ed., Dover Publication: New York, 1961.

[16] Palermo L., Jr., The tangential differential operator applied to a stress boundary integral equation for plate bending including the shear deformation effect. Engineering Analysis with Boundary Elements, 36, pp. 1213-1225, 2012.

[17] Wrobel, L.C., The Boundary Element Method: Applications in Thermo-Fluids and Acoustics, John Wiley \& Sons Ltd.: Chichester, 2002. 
[18] Telles, J.C.F., A self-adaptive coordinate transformation for efficient numerical evaluation of general boundary element integrals. International Journal for Numerical Methods in Engineering, 24, pp. 959-973, 1987.

[19] Wilkinson, J.H., The Algebraic Eigenvalue Problem, Oxford University Press, William Clowes \& Sons: London, 1972. 